\title{
Measurements of Li Abundance in a sample of T Tauri Stars
}

\author{
M. J. Sartori, J. Gregorio-Hetem, B. V. Castilho and J. R. D. Lépine \\ Universidade de São Paulo, CP 3386, São Paulo, SP, 01060-970, Brazil
}

\begin{abstract}
In this work we present the stellar parameters and the $\mathrm{Li}$ abundances, obtained by spectral synthesis, for a sample of 13 weak-line $\mathrm{T}$ Tauri stars.
\end{abstract}

\section{Stellar Properties}

In the frame of a study of kinematics and stellar properties of the pre-main sequence population of southern star forming regions, we are analyzing the evolutionary status of a sample T Tauri (TT) stars, on the basis of their stellar properties compared to their kinematics. In this work we studied 13 weak-line TT (WTT) stars of the Chamaeleon, Lupus, Ophiuchus and Upper Scorpius star-forming regions.

The absolute bolometric luminosities were estimated combining spectral types (S.T.), V and R with the distance of the associations, assuming intrinsic colours and bolometric corrections from Bessel, Castelli, \& Plez (1998), and a S.T.- $T_{\text {eff }}$ relation from de Jager \& Nieuwenhuijzen (1987). The S.T. adopted were taken from literature (see Notes in Table 1) and from this work. For the star HT Lup the luminosity was calculated using the distance obtained from the HIPPARCOS parallax. The star WRA 488 is an isolated TT and does not have distance estimation. The ages and masses (see Table 1) were determined by comparison with D'Antona \& Mazzitelli (1994) evolutionary tracks and isochrones which use CM convection and Alexander opacities.

\section{Lithium Abundances}

We obtained medium-resolution $(\mathrm{R}=7500)$ Coudé spectra in the region of the Li I line at $\lambda 6708 \AA$, using the $1.6 \mathrm{~m}$ telescope at Pico dos Dias Observatory (MG, Brazil).

$\mathrm{Li}$ abundances were estimated comparing the observed spectra to LTE synthetic spectra computed with the code SPECTRUM (kindly made available by R. O. Gray) and Kurucz's (1992) stellar atmosphere models. The errors in $\log \mathrm{N}(\mathrm{Li})$ are mainly due to the $T_{\text {eff }}$ uncertainty, and are $\approx 0.3 \mathrm{dex}$. Our results are consistent with the Li depletion predicted by Mendes, D'Antona \& Mazzitelli (1999) for $1.0,0.8$ and $0.6 M_{\odot}$ stars. 
Table 1. Stellar parameters and Li abundances of WTT stars

\begin{tabular}{llcccccc}
\hline Object & S.T. & $\begin{array}{c}\mathrm{L} \\
\left(\mathrm{L}_{\odot}\right)\end{array}$ & $\begin{array}{c}\text { Age } \\
(\mathrm{Myr})\end{array}$ & $\begin{array}{c}\text { Mass } \\
\left(M_{\odot}\right)\end{array}$ & $\begin{array}{c}W_{\lambda}(\mathrm{Li}) \\
(\mathrm{m} \AA)\end{array}$ & $\operatorname{logN}(\mathrm{Li})$ & Notes \\
\hline WRA 488 & G6 V & & & & 199 & 3.0 & $\mathrm{a}, \mathrm{i}^{*}$ \\
CHXR 3 & K0 V & 1.77 & 7.0 & 1.45 & 59 & $\leq 2.0$ & $\mathrm{~b}, \mathrm{e}$ \\
CHXR 8 & G2 V & 0.47 & $>100$ & 0.9 & 134 & 3.0 & $\mathrm{~b}, \mathrm{i}$ \\
CHXR 11 & G8 V & 2.52 & 7.0 & 1.6 & 135 & 2.3 & $\mathrm{~b}, \mathrm{i}$ \\
CHX 18N & K2 V & 0.70 & 15.0 & 1.1 & 534 & 3.5 & $\mathrm{~b}, \mathrm{i}$ \\
Sz 41 & K2 V & 0.73 & 10.0 & 1.1 & 454 & 3.5 & $\mathrm{a}, \mathrm{g}$ \\
CoD-40 8434 & K5 V & 0.55 & 7.0 & 0.9 & 504 & 3.5 & $\mathrm{a}, \mathrm{g}$ \\
HT Lup & K2 V & 3.95 & 0.7 & 1.1 & 472 & 3.5 & $\mathrm{a}, \mathrm{f}$ \\
Wa Oph/1 & K2 IV & 1.10 & 2.5 & 0.8 & 482 & 3.3 & $\mathrm{c}, \mathrm{h}$ \\
Wa Oph/3 & K0 IV & 1.91 & 2.5 & 1.1 & 534 & 3.4 & $\mathrm{c}, \mathrm{h}$ \\
V896 Sco & K7 V & 0.90 & 1.5 & 0.6 & 520 & 3.1 & $\mathrm{a}, \mathrm{g}$ \\
CoD-24 12809 & K3 V & 1.37 & 3.0 & 1.0 & 529 & 3.6 & $\mathrm{a}, \mathrm{i}$ \\
Wa CrA/2 & G5 IV & 1.09 & 20.0 & 1.2 & 312 & 3.2 & $\mathrm{~d}, \mathrm{~h}$ \\
\hline
\end{tabular}

Notes: (V-R) and S.T. from: (a) Gregorio-Hetem et al. (1992), (b) Lawson, Feigelson, \& Huenemoerder (1996), (c) Walter et al. 1994, (d) Walter et al. 1997, (e) Alcalá (1994), (f) Herbig (1977), (g) Torres (1998), (h) Walter (1986), (i) this work. (*) Isolated TT star with no distance determination.

\section{References}

Alcalá, J. M. 1994, PhD Thesis, Ruprecht-Karls-Univ., Heidelberg

Bessel, M. S., Castelli, F., \& Plez, B. 1998, A\&A, 333, 231

D'Antona, F., \& Mazzitelli, I. 1994, ApJS, 90, 467

de Jager, C., \& Nieuwenhuijzen, H. 1987, A\&A, 177, 217

Gregorio-Hetem, J., Lépine, J. R. D., Quast, G. R., Torres, C. A. O., \& de la Reza, R. 1992, AJ, 103, 549

Herbig, G. H. 1977, ApJ, 214, 747

Kurucz, R. L. 1992, in IAU Symp. 149, ed. B. Barbuy \& A. Renzini (Kluwer Acad. Press), 255

Lawson, W. A., Feigelson E. D., \& Huenemoerder, D. P. 1996, MNRAS, 280, 1071

Mendes, L. T. S., D’Antona, F., \& Mazzitelli, I. 1999, A\&A, 341, 174

Torres, C. A. O. 1998, PhD Thesis, Observatório Nacional, Brazil

Walter, F. M. 1986, ApJ, 306, 573

Walter, F. M., Vrba, F. J., Mathieu, R. D., Brown, A., \& Myers, P. C. 1994, AJ, 107, 692

Walter, F. M., Vrba, F. J., Wolk, S. J., Mathieu, R. D., \& Neuhäuser, R. 1997, AJ, 114, 1544 